\title{
MESH-FREE ANALYSIS OF PLATE BENDING PROBLEMS BY MOVING FINITE ELEMENT APPROXIMATION
}

\author{
VLADIMIR SLADEK, JAN SLADEK \& MIROSLAV REPKA \\ Institute of Construction and Architecture, Slovak Academy of Sciences, Slovakia
}

\begin{abstract}
The governing equations for plate bending problems are given by the partial differential equations of the 4th order. Therefore $\mathrm{C}^{1}$ continuous elements are required for approximation and discretization with using the standard finite element method (FEM). A novel discretization method is proposed and developed for numerical solution of plate bending boundary value problems. In contrast to the standard FEM, the analysed domain is not covered by a mesh of fixed non-overlapping finite elements, but only a net of nodes is used for discretization. Around each node, there is properly created a Lagrange finite element and the spatial variation of field variables is interpolated within this element in terms of nodal values and polynomial shape functions defined in the intrinsic coordinate space of the finite element. Thus, the Lagrange finite element associated with a node is moving within the analysed domain from node to node. Since there are no element interfaces known in standard FEM with a fixed finite element mesh, the difficulties with continuity of derivatives of field variables on such interfaces are avoided and higher order derivatives are available within the moving finite element. This makes the moving finite element (MFE) approximation to be applicable also to the development of the strong formulation of a boundary value problem with collocation of both the governing equations and boundary conditions at interior and boundary nodes, respectively. To decrease the order of the polynomial interpolation, the original set of the governing PDE is decomposed into a system of 2nd order PDEs by introducing a new field variable. Then, the boundary conditions are to be modified and the bi-quadratic Lagrange finite element is sufficient for approximation. Both the strong and the local weak formulations are derived and employed in the numerical test examples with focusing on verification of the reliability (accuracy and stability) and efficiency of the new method.
\end{abstract}

Keywords: Lagrange finite element, strong and weak formulation, convergence and accuracy, numerical stability, computational efficiency.

\section{INTRODUCTION}

The most frequently used methods in engineering computations, such as finite element method (FEM), finite volume method (FVM), boundary element method (BEM) are based on weak formulations of boundary value problems. Although, the strong formulations offer better computational efficiency because of elimination of integrations, the price which should be paid is the need to approximate higher order derivatives. The standard finite elements (FE) give only $\mathrm{C}^{0}$ continuity, while the recently developed mesh-free approximations utilize the higher order continuous shape functions, but these are not expressed in terms of elementary functions and hence, such mesh-free approximations result into time consuming evaluation of shape functions. Utilization of advantages of the polynomial interpolation (like in FEM) and the element-free discretization of the analysed domain (like in mesh-free methods) has been proposed originally in works [1]-[3] and recently elaborated in [4]-[6]. In this paper, the moving finite element (MFE) approximation [4] is developed and implemented for the solution of plate bending boundary value problems, with studying numerical aspects of the proposed method. 


\section{FORMULATION OF BOUNDARY VALUE PROBLEMS FOR THIN ELASTIC PLATES}

The bending of elastic plates can be described in a unique way [7] by including the assumptions of three plate bending theories, such as the Kirchhoff-Love theory (KLT), shear deformation plate bending theories of the 1st and 3rd order (FSDPT and TSDPT). Considering the thin elastic, homogeneous plates, the tensor of bending moments is expressed in terms of derivatives of dimensionless deflections $w^{*}(\mathbf{x})=w(\mathbf{x}) / h$ as

$$
M_{\alpha \beta}^{*(w)}=(1-v) w_{, \alpha \beta}^{*}+v \delta_{\alpha \beta} \nabla^{2} w^{*}, \quad M_{\alpha \beta}^{*(w)}(\mathbf{x})=\frac{L^{2}}{D h} M_{\alpha \beta}^{(w)}(\mathbf{x}),
$$

where $h, v, D, L$ are the plate thickness, Poisson ratio, bending stiffness, a characteristic length in the mid-plane domain $\Omega$, respectively, and the superscript * stands for notation of dimensionless quantities. The dimensionless in-plane Cartesian coordinates are defined as $x_{\alpha}^{*}=x_{\alpha} / L$, and the superscript will be omitted in Cartesian coordinates in what follows, because we shall deal only with the dimensionless coordinates. The governing equation of the plate subjected to transversal loading $t_{3}(\mathbf{x})$ is given by

$$
M_{\alpha \beta, \alpha \beta}^{*(w)}=-t_{3}^{*}, \quad t_{3}^{*}=\frac{L^{4}}{D h} t_{3},
$$

and can be rewritten as

$$
\nabla^{2} \nabla^{2} w^{*}=t_{3}^{*}
$$

In order to decrease the order of the derivatives of deflection field, it is convenient to introduce the additional field variable $m^{*}$ by eqn (4), when the 4th order PDE (3) is replaced by the system of two 2nd order PDE

$$
\nabla^{2} w^{*}-m^{*}=0, \quad \nabla^{2} m^{*}=t_{3}^{*} .
$$

The homogeneous boundary conditions can be written on the plate edge as

(i) clamped edge $(\mathrm{CE})$ :

$$
\left.w^{*}\right|_{\partial \Omega}=0, \quad \partial w^{*} /\left.\partial \mathbf{n}\right|_{\partial \Omega}=0
$$

(ii) simply supported edge (SSE):

$$
\left.w^{*}\right|_{\partial \Omega}=0,\left.\quad n_{\alpha} n_{\beta} M_{\alpha \beta}^{*(w)}\right|_{\partial \Omega}=0
$$

(iii) free edge (FrE):

$$
\left.n_{\alpha} n_{\beta} M_{\alpha \beta}^{*(w)}\right|_{\partial \Omega}=0,\left.\quad V^{*}\right|_{\partial \Omega}=0
$$

with 


$$
\begin{aligned}
V^{*}(\mathbf{x}) & :=n_{\alpha}(\mathbf{x}) M_{\alpha \beta, \beta}^{(w) *}(\mathbf{x})+\frac{\partial T^{(w) *}}{\partial \mathbf{t}}(\mathbf{x})-\sum_{c} \llbracket T^{(w) *}\left(\mathbf{x}^{c}\right) \rrbracket \delta\left(\mathbf{x}-\mathbf{x}^{c}\right) \\
& =n_{\alpha}(\mathbf{x}) m_{, \alpha}^{*}(\mathbf{x})+\frac{\partial T^{(w) *}}{\partial \mathbf{t}}(\mathbf{x})-\sum_{c} \llbracket T^{(w) *}\left(\mathbf{x}^{c}\right) \rrbracket \delta\left(\mathbf{x}-\mathbf{x}^{c}\right),
\end{aligned}
$$

where $T^{(w) *}=\frac{L^{2}}{D h} T^{(w)}, T^{(w)}:=t_{\alpha} n_{\beta} M_{\alpha \beta}^{(w)}$ is the twisting moment, and the jump term is defined at corner points $\mathbf{x}^{c}$ on the boundary edge $\partial \Omega$ as

$$
\llbracket T^{(w)}\left(\mathbf{x}^{c}\right) \rrbracket:=T^{(w)}\left(\mathbf{x}^{c}-0\right)-T^{(w)}\left(\mathbf{x}^{c}+0\right) .
$$

Recall that at corner points the $\partial w^{*} /\left.\partial \mathbf{n}\right|_{\partial \Omega}$ is not defined uniquely because of the discontinuity of the normal vector. Therefore it is convenient to replace this boundary condition at $\mathbf{x}^{c}$ by the local weak form of the governing eqn $\left(4_{1}\right)$

$$
\int_{\Omega^{c}}\left(\nabla^{2} w^{*}-m^{*}\right) d \Omega=0 \Rightarrow \int_{L^{c}} n_{\alpha} w_{, \alpha}^{*} d \Gamma-\int_{\Omega^{c}} m^{*} d \Omega=0
$$

on the local subdomain around the point $\mathbf{x}^{c}$, where we have utilized that the boundary of the subdomain $\Omega^{c}$ is $\partial \Omega^{c}=\Gamma^{c} \cup L^{c}$ and $\partial w^{*} /\left.\partial \mathbf{n}\right|_{\Gamma^{c}}=0$.

In view of (1) the boundary condition

$$
\left.n_{\alpha} n_{\beta} M_{\alpha \beta}^{*(w)}\right|_{\partial \Omega}=0
$$

can be rewritten as $\left.\left[\nabla^{2} w^{*}-(1-v) t_{\alpha} t_{\beta} w_{, \alpha \beta}^{*}\right]\right|_{\partial \Omega}=0$. Furthermore,

$t_{\alpha} t_{\beta} w_{, \alpha \beta}^{*}=\frac{\partial}{\partial \mathbf{t}}\left(t_{\beta} w_{, \beta}^{*}\right)-\frac{\partial t_{\beta}}{\partial \mathbf{t}} w_{, \beta}^{*}=\frac{\partial}{\partial \mathbf{t}}\left(\frac{\partial w^{*}}{\partial \mathbf{t}}\right)-\frac{\partial t_{\beta}}{\partial \mathbf{t}}\left(n_{\beta} n_{\gamma}+t_{\beta} t_{\gamma}\right) w_{, \gamma}^{*}=\frac{\partial}{\partial \mathbf{t}}\left(\frac{\partial w^{*}}{\partial \mathbf{t}}\right)-\kappa \frac{\partial w^{*}}{\partial \mathbf{n}}$ with $\kappa=n_{\beta} \frac{\partial t_{\beta}}{\partial \mathbf{t}}$ being the curvature of the boundary edge. If $\left.w^{*}\right|_{\partial \Omega}=0$, then $\partial w^{*} /\left.\partial \mathbf{t}\right|_{\partial \Omega}=0$ as well as $\partial\left(\partial w^{*} / \partial \mathbf{t}\right) /\left.\partial \mathbf{t}\right|_{\partial \Omega}=0$ and the boundary condition (7) on the SSE becomes $\left.\left[\nabla^{2} w^{*}+\kappa \partial w^{*} / \partial \mathbf{n}\right]\right|_{\partial \Omega}=0$. Eventually, the boundary conditions on the SSE can be rewritten as

$$
\left.w^{*}\right|_{\partial \Omega}=0,\left.\quad\left[m^{*}+\kappa \partial w^{*} / \partial \mathbf{n}\right]\right|_{\partial \Omega}=0
$$


The advantage of this expression of the boundary conditions is the greater convenience for numerical treatment than the expression given by $(5 \mathrm{~b})$ because of the lower order of the derivatives involved. The curvature is vanishing on straight edges as well as at corners, if the edges on both sides of the corner are straight lines. Then, the boundary condition $\left(8_{2}\right)$ is simplified as $\left.m^{*}\right|_{\partial \Omega}=0$ and both the boundary conditions on SSE are Dirichlet type. Nevertheless, a special treatment is required at corner points, in general. One possibility is to consider $\left(8_{2}\right)$ in weak sense on the very short segments $\Gamma^{c s}$ and $\Gamma^{c f}$ of the length $\varepsilon$. Then,

$$
\int_{\Gamma^{c s} \cup \Gamma^{c f}}\left[m^{*}+\kappa \partial w^{*} / \partial \mathbf{n}\right] d \Gamma \approx 2 \varepsilon m^{*}\left(\mathbf{x}^{c}\right)+\varepsilon\left(\kappa\left(\mathbf{x}^{c s}\right) \frac{\partial w^{*}}{\partial \mathbf{n}}\left(\mathbf{x}^{c s}\right)+\kappa\left(\mathbf{x}^{c f}\right) \frac{\partial w^{*}}{\partial \mathbf{n}}\left(\mathbf{x}^{c f}\right)\right)=0,
$$

and finally, the boundary conditions at a corner on the SSE can be rewritten as

$$
\left.w^{*}\right|_{\partial \Omega}=0, \quad m^{*}\left(\mathbf{x}^{c}\right)+\frac{1}{2}\left(\kappa\left(\mathbf{x}^{c s}\right) \frac{\partial w^{*}}{\partial \mathbf{n}}\left(\mathbf{x}^{c s}\right)+\kappa\left(\mathbf{x}^{c f}\right) \frac{\partial w^{*}}{\partial \mathbf{n}}\left(\mathbf{x}^{c f}\right)\right)=0 .
$$

In the case of free-edge, it is necessary to rearrange the boundary condition $\left.V^{*}\right|_{\partial \Omega}=0$. Owing to the Dirac-delta function as well as the discontinuity of the normal vector at a corner point, it is necessary to consider this boundary condition in weak sense around arbitrary point on the boundary edge, i.e. in weak sense on $\Gamma^{c s} \cup \Gamma^{c f}$ and/or $\Gamma^{b s} \cup \Gamma^{b f}$ considered around $\mathbf{x}^{c}$ and/or $\mathbf{x}^{b}$, respectively (see Fig. 1). For the sake of brevity, we shall use the superscript " $b$ " for arbitrary point on the boundary edge. Then, the weak form of the considered boundary condition takes the form

$$
\begin{aligned}
\int_{\Gamma^{b s} \cup \Gamma^{b f}}\left[n_{\alpha} m_{, \alpha}^{*}+\frac{\partial T^{(w) *}}{\partial \mathbf{t}}-\sum_{c} \llbracket T^{(w) *}\left(\mathbf{x}^{c}\right) \rrbracket \delta\left(\mathbf{x}-\mathbf{x}^{c}\right)\right] d \Gamma & =\int_{\Gamma^{b s} \cup \Gamma^{b f}} n_{\alpha} m_{, \alpha}^{*} d \Gamma+ \\
& +T^{(w) *}\left(\mathbf{x}^{b f}\right)-T^{(w) *}\left(\mathbf{x}^{b s}\right) .
\end{aligned}
$$

Since on short segments of the boundary edge, we can write

$$
\begin{aligned}
& \int_{\Gamma^{b s} \cup \Gamma^{b f}} n_{\alpha} m_{, \alpha}^{*} d \Gamma \approx \varepsilon\left(\frac{\partial m^{*}}{\partial \mathbf{n}}\left(\mathbf{x}^{b s}\right)+\frac{\partial m^{*}}{\partial \mathbf{n}}\left(\mathbf{x}^{b f}\right)\right) \text {, the b.c. }\left.V^{*}\right|_{\partial \Omega}=0 \text { becomes } \\
& T^{(w) *}\left(\mathbf{x}^{b f}\right)-T^{(w) *}\left(\mathbf{x}^{b s}\right)+\varepsilon\left(\frac{\partial m^{*}}{\partial \mathbf{n}}\left(\mathbf{x}^{b s}\right)+\frac{\partial m^{*}}{\partial \mathbf{n}}\left(\mathbf{x}^{b f}\right)\right)=0 .
\end{aligned}
$$

This expression of boundary condition still involves the 2 nd order derivatives in twisting moment terms.

In numerical solutions of boundary value problems, the governing equations can be considered either in the strong sense at interior nodes $\mathbf{x}^{a} \in \Omega$ as

$$
\nabla^{2} w^{*}\left(\mathbf{x}^{a}\right)-m^{*}\left(\mathbf{x}^{a}\right)=0, \quad \nabla^{2} m^{*}\left(\mathbf{x}^{a}\right)=t_{3}^{*}\left(\mathbf{x}^{a}\right),
$$



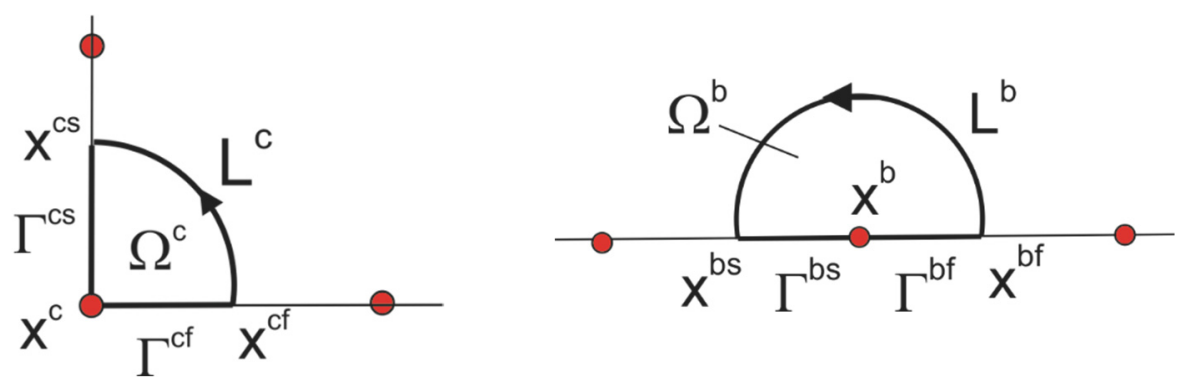

Figure 1: Illustration of boundary contours $\Gamma^{c}=\Gamma^{c s} \cup \Gamma^{c f}$ and $L^{c}$ associated with a corner point $\mathbf{x}^{c}$ or a boundary node $\mathbf{x}^{b}$ on a smooth portion of the boundary.

or in the local weak sense on small subdomain $\Omega^{a}{ }_{\ni} \mathbf{x}^{a}$ around interior nodes $\mathbf{x}^{a} \in \Omega$ as

$$
\int_{L^{a}} n_{\alpha} w_{, \alpha}^{*} d \Gamma-\int_{\Omega^{a}} m^{*} d \Omega=0, \quad \int_{L^{a}} n_{\alpha} m_{, \alpha}^{*} d \Gamma-\int_{\Omega^{a}} t_{3}^{*} d \Omega=0 .
$$

It is seen that the 2 nd order derivatives of primary field variables are required in the case strong formulation and the 1st order derivatives are sufficient in the weak formulation. On the other hand, these derivatives are needed only at nodal point in case of the strong formulation, while they are needed at certain integration points in case of weak formulation. Thus, the strong formulation requires higher order continuity of approximation than the weak formulation, while the computational efficiency is worse in the case of weak formulation.

\section{MOVING FINITE ELEMENT APPROXIMATION}

In contrast to the classical FEM, the analysed domain is not discretized into the mesh of finite elements, but only a mesh of nodes is utilized [4]. A finite element is associated with each node and this element is created automatically according to the position of the reference node on the bounded domain $\Omega \cup \partial \Omega$ [4]. In this paper, we shall consider the bi-quadratic Lagrange elements (with 9 nodes). Construction of the moving finite elements (FE) associated with particular nodes $\mathbf{x}^{a}$ follows the rules [4]: (i) if $\mathbf{x}^{a} \in \Omega, \mathbf{x}^{a}$ is the centre of the FE; (ii) if $\mathbf{x}^{a} \in \partial \Omega$ and $\partial \Omega$ is smooth at the node $\mathbf{x}^{a}$, this node is the mid-side node of the FE; (iii) if $\mathbf{x}^{a}$ lies at a corner on the boundary edge $\partial \Omega$, the FE is constructed uniquely. The approximation of the spatial variation of primary field variables, such as $u(\mathbf{x}) \in\left\{w^{*}(\mathbf{x}), m^{*}(\mathbf{x})\right\}$, is given by polynomial interpolation within the moving finite element

$$
E^{a} \quad \text { as }\left.\quad u(\mathbf{x})\right|_{E^{a}} \approx \sum_{\gamma=1}^{9} u^{a_{\gamma}} N^{\gamma}(\xi), \quad u^{a_{\gamma}}=u\left(\mathbf{x}^{a_{\gamma}}\right),
$$

where $\xi$ is the shortcut for intrinsic coordinates $\left(\xi_{1}, \xi_{2}\right), N^{\gamma}(\xi)$ stand for standard interpolation shape functions in Lagrange finite element [9], and $a_{\gamma}$ is the global number of the node on the element $E^{a}$ with the local number $\gamma \in\{1,2, \ldots, 9\}$. Utilization of bi-quadratic Lagrange FE for interpolation enables us to apply also the formulations involving the 2nd 
order derivatives of field variables. Since the polynomial interpolation is in terms of intrinsic coordinates, the expression of derivatives is slightly complicated. Therefore we give them, but without details of derivation. The gradient of the field variable is given within the moving $\mathrm{FE}$ as

$$
\left.u_{, i}(\mathbf{x})\right|_{E^{a}}=\left.\frac{\partial u(\mathbf{x})}{\partial x_{i}}\right|_{E^{a}} \approx \sum_{\gamma=1}^{9} u^{a_{\gamma}} b_{i}^{\gamma a}(\boldsymbol{\xi}), \quad b_{i}^{\gamma a}(\xi)=\mathrm{Y}_{i k}^{a} \frac{\partial N^{\gamma}(\boldsymbol{\xi})}{\partial \xi_{k}}, \quad \mathrm{Y}_{i k}^{a}=\left.\frac{\partial \xi_{k}}{\partial x_{i}}\right|_{E^{a}},
$$

in which $\left[Y^{a}\right]=\left[J^{a}\right]^{-1}$ and

$$
J_{k i}^{a}=\left.\frac{\partial x_{i}}{\partial \xi_{k}}\right|_{E^{a}}=\sum_{\gamma=1}^{9} x_{i}^{a_{\gamma}} \frac{\partial N^{\gamma}}{\partial \xi_{k}}, \quad Y_{i j}^{a}=\frac{\varepsilon_{i k 3} \varepsilon_{j l 3}}{\left|J^{a}\right|} J_{l k}^{a}, \quad\left|J^{a}\right|=\varepsilon_{m n 3} J_{1 m}^{a} J_{2 n}^{a},
$$

since we have used the isoparametric elements for field variables and Cartesian coordinates

$$
\left.x_{i}\right|_{E^{a}} \approx \sum_{\gamma=1}^{9} x_{i}^{a_{\gamma}} N^{\gamma}(\xi)
$$

Similarly, for the 2nd order derivatives, we obtain

$$
\begin{aligned}
& \left.u_{, i j}(\mathbf{x})\right|_{E^{a}}=\left.\frac{\partial^{2} u(\mathbf{x})}{\partial x_{i} \partial x_{j}}\right|_{E^{a}} \approx \sum_{\gamma=1}^{n} u^{a_{\gamma}} \frac{\partial \xi_{m}}{\partial x_{j}} \frac{\partial}{\partial \xi_{m}} b_{i}^{\gamma a}(\boldsymbol{\xi}) \\
= & \sum_{\gamma=1}^{n} u^{a_{\gamma}} Y_{j m}^{a}\left[Y_{i k}^{a} N_{, k m}^{\gamma}(\boldsymbol{\xi})-Y_{i s}^{a} J_{s l, m}^{a} b_{l}^{\gamma a}(\boldsymbol{\xi})\right]=\sum_{\gamma=1}^{n} u^{a_{\gamma}} b_{i j}^{\gamma a}(\boldsymbol{\xi}),
\end{aligned}
$$

with $J_{s l, m}^{a}=\sum_{\gamma=1}^{n} x_{l}^{a_{\gamma}} \frac{\partial^{2} N^{\gamma}}{\partial \xi_{s} \partial \xi_{m}}=J_{m l, \mathrm{~s}}^{a}$,

$$
b_{i j}^{\gamma a}(\xi):=Y_{j m}^{a}\left[Y_{i k}^{a} N_{, k m}^{\gamma}(\xi)-Y_{i s}^{a} J_{s l, m}^{a} b_{l}^{\gamma a}(\xi)\right]=b_{j i}^{\gamma a}(\xi) .
$$

The numerical implementation of the strong formulation can be obtained very easily by using the expressions for the derivatives at nodal points in both the governing equations and boundary conditions. In the case of weak formulation, some additional implementation is needed for the geometry of local subdomains and boundary contours, and it can be done as shown in [4]. There is no restriction on the shape of the local subdomain around a nodal point, but the subdomain should lie within the moving FE associated with the considered nodal point. For numerical integrations, the most appropriate shape of subdomain is the circular subdomain in the intrinsic coordinate space.

\section{NUMERICAL EXAMPLES}

In order to test the accuracy, numerical stability and compare the computational efficiency, we have considered simple examples for bending of a thin elastic homogeneous plate with clamped and/or simply supported edges and subjected to stationary uniform transversal 
loading $t_{3}^{*}=1$. The square plate $L \times L$ is considered with the thickness $h=L / 50$ and constant Poisson ratio $v=0.3$. For the governing equations (g.e.), we have used two alternatives - the strong as well as weak formulations, while the Dirichlet boundary conditions are considered in strong sense and the Neumann boundary conditions either in strong or weak sense. The convergence study with increasing the number of uniformly distributed nodal points is shown in Figs 2 and 3.

Practically the same results have been obtained for all the formulation variants applied to plate with CE: (a) weak form of g.e. and Neumann b.c. at all boundary nodes, (b) weak form of g.e. and Neumann b.c. only at corners, (c) strong form of g.e. and weak form of Neumann b.c. at all nodes. Convergence is achieved for rather large amount of nodes $(51 \times 51=2601)$.

In case of the plate with SSE, the convergence is significantly faster $(11 \times 11=121$ nodes $)$ and it is practically the same in both the considered formulation variants: (a) weak form of g.e., (b) strong form of g.e. The boundary conditions in plates with SSE are Dirichlet type and considered only in the strong sense.

From comparison of Figs 2 and 3, it is seen that the vanishing slope (normal derivative of deflections) on the CE affects significantly also the values of deflections at nodes far from the boundary edge, if the approximation elements are extensively large.

Hence, the convergence is much slower than in case of the plate with SSE. Moreover, in case of SSE plate, the analytical solution is available in the form of infinite series [8], which converges fast. On the other hand, the closed form solution is not available for CE plate. Therefore, we can present comparison of numerically obtained distribution of deflections with analytical one only for the SSE plate (Fig. 4).

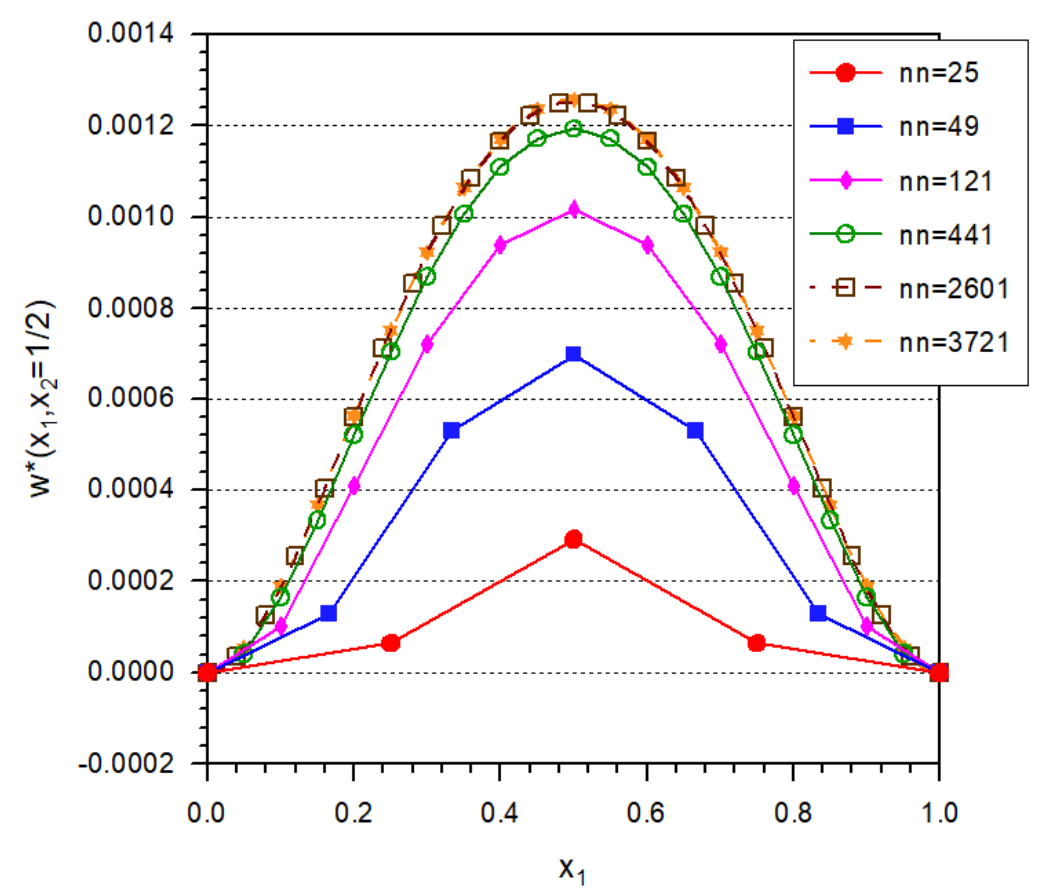

Figure 2: Convergence of numerical results for deflections in plate with CE. 


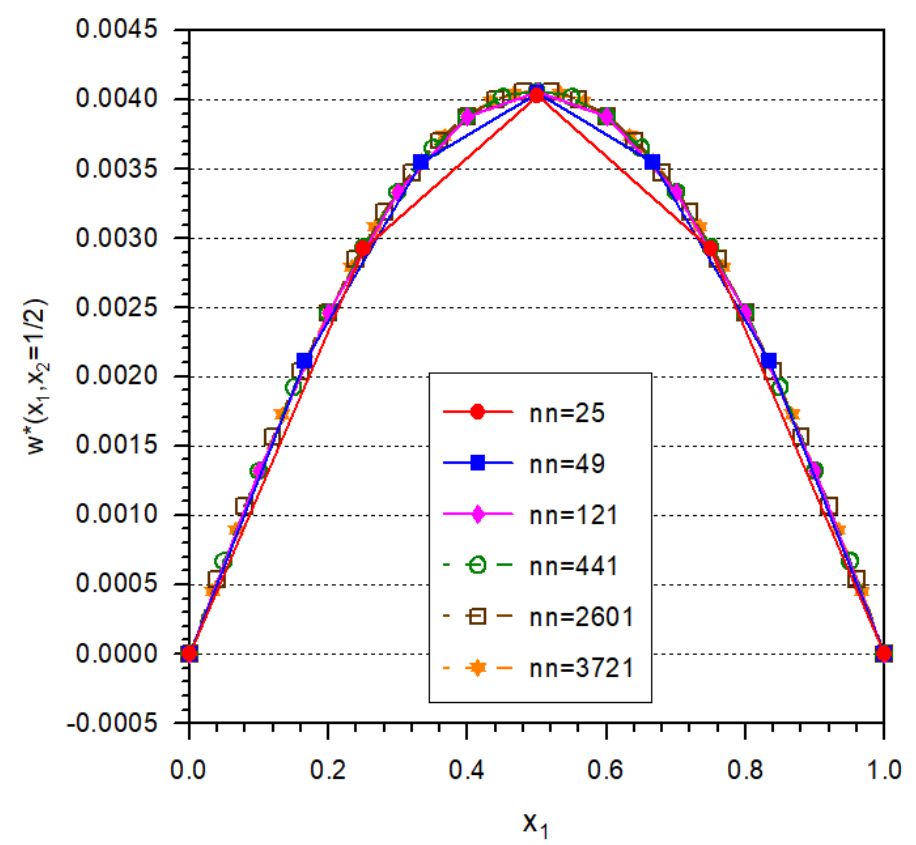

Figure 3: Convergence of numerical results for deflections in plate with SSE.

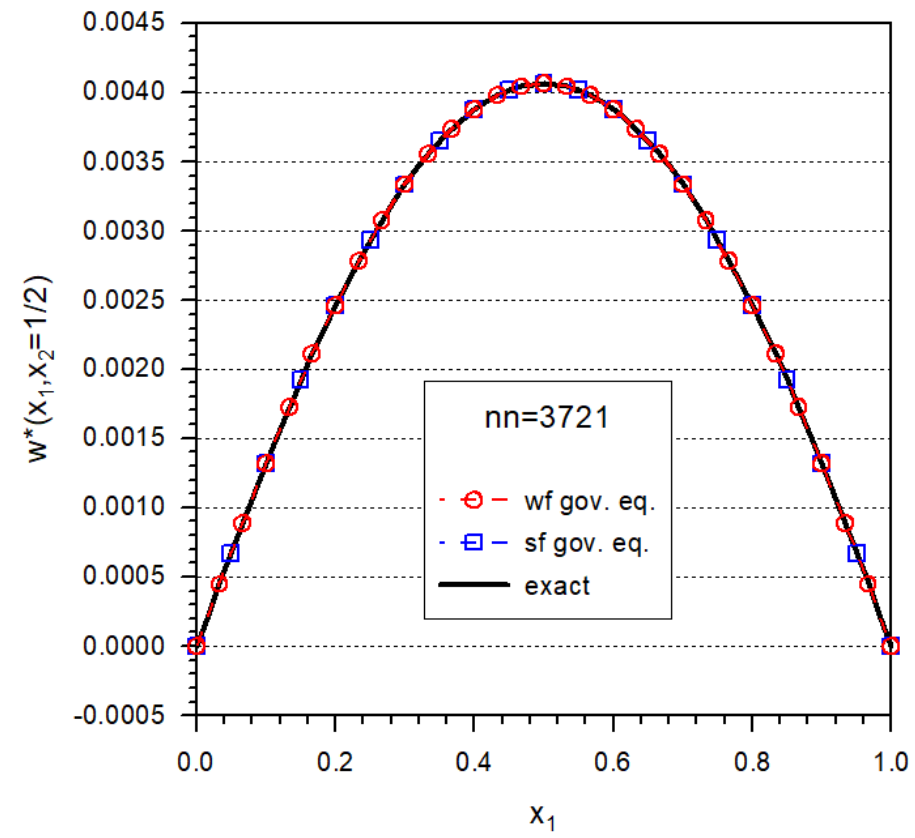

Figure 4: Comparison of numerically computed distribution of deflections with analytical one in SSE plate. 
The deviation of numerically computed maximal deflection $w_{\max }^{*}=0.4062227 \times 10^{-2} q^{*}$ from the exact value $w_{\max }^{*}=0.4062353 \times 10^{-2} q^{*}$ is $0.0031 \%$ in case of the SSE plate, while in the CE plate the numerical value $w_{\max }^{*}=0.1258785 \times 10^{-2} q^{*}$ deviates from the exact value $w_{\max }^{*}=0.126 \times 10^{-2} q^{*}$ by $0.096 \%$, provided that 5041 nodes were used in numerical computations.

Furthermore, for the SSE plate, we can evaluate also the average error as

$$
\% \text { error of deflections }=\frac{1}{n n} \sum_{a=1}^{n n}\left|\frac{w^{*}\left(\mathbf{x}^{a}\right)}{w_{e x}^{*}\left(\mathbf{x}^{a}\right)}-1\right| \times 100 \%,
$$

and the convergence of the computational method can be measured via the dependence of the $\%$ error of deflections on the number of nodes ( $n n)$ (Fig. 5). The exact solution for the SSE plate [8] is given as

$$
w_{e x}^{*}=\frac{16}{\pi^{6}} \frac{t_{3}^{*}}{L^{4}} \sum_{m=1,3, \ldots n=1,3, \ldots}^{\infty} \sum_{m n\left[\left(\frac{m}{L_{1}}\right)^{2}+\left(\frac{n}{L_{2}}\right)^{2}\right]^{2}}^{\infty}, \quad L_{1}=L_{2}=L .
$$

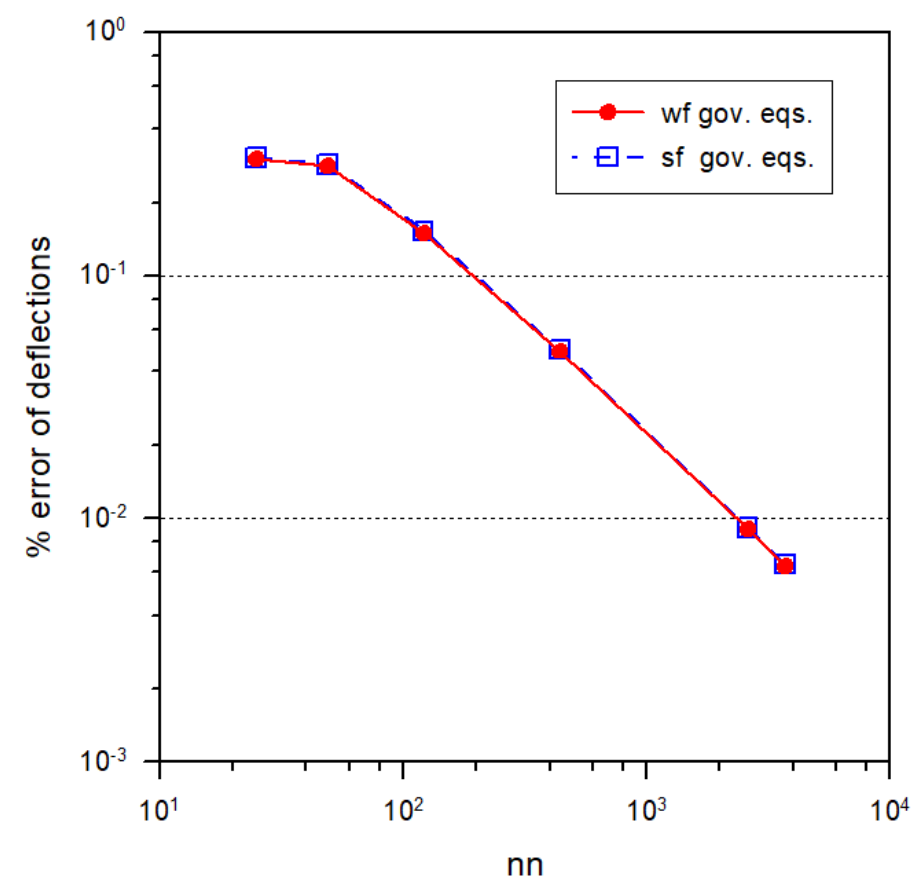

Figure 5: Dependence of the \% error of deflections on the number of nodes in SSE plate. 

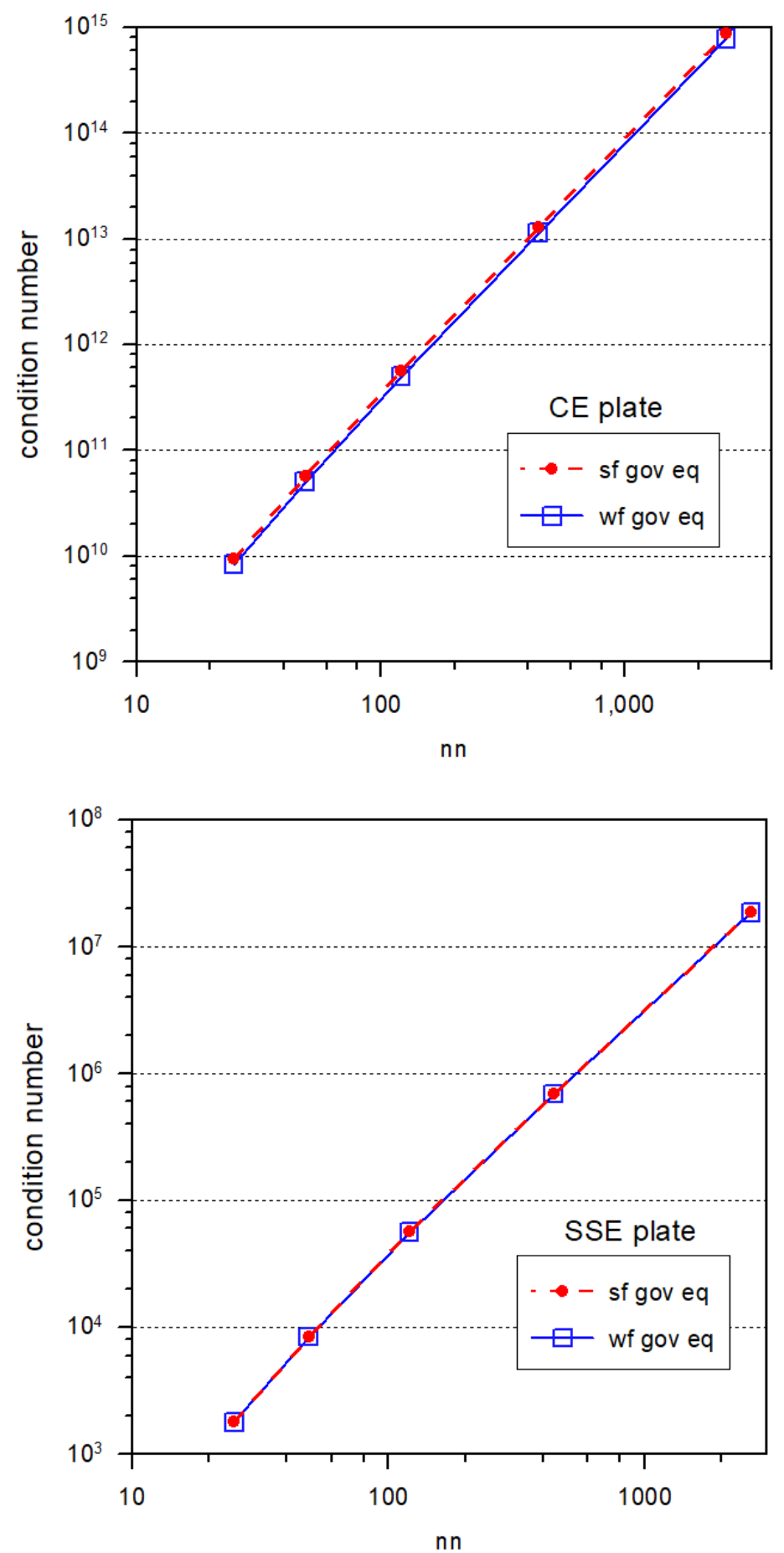

Figure 6: Condition number of discretized equations vs. number of nodes. 

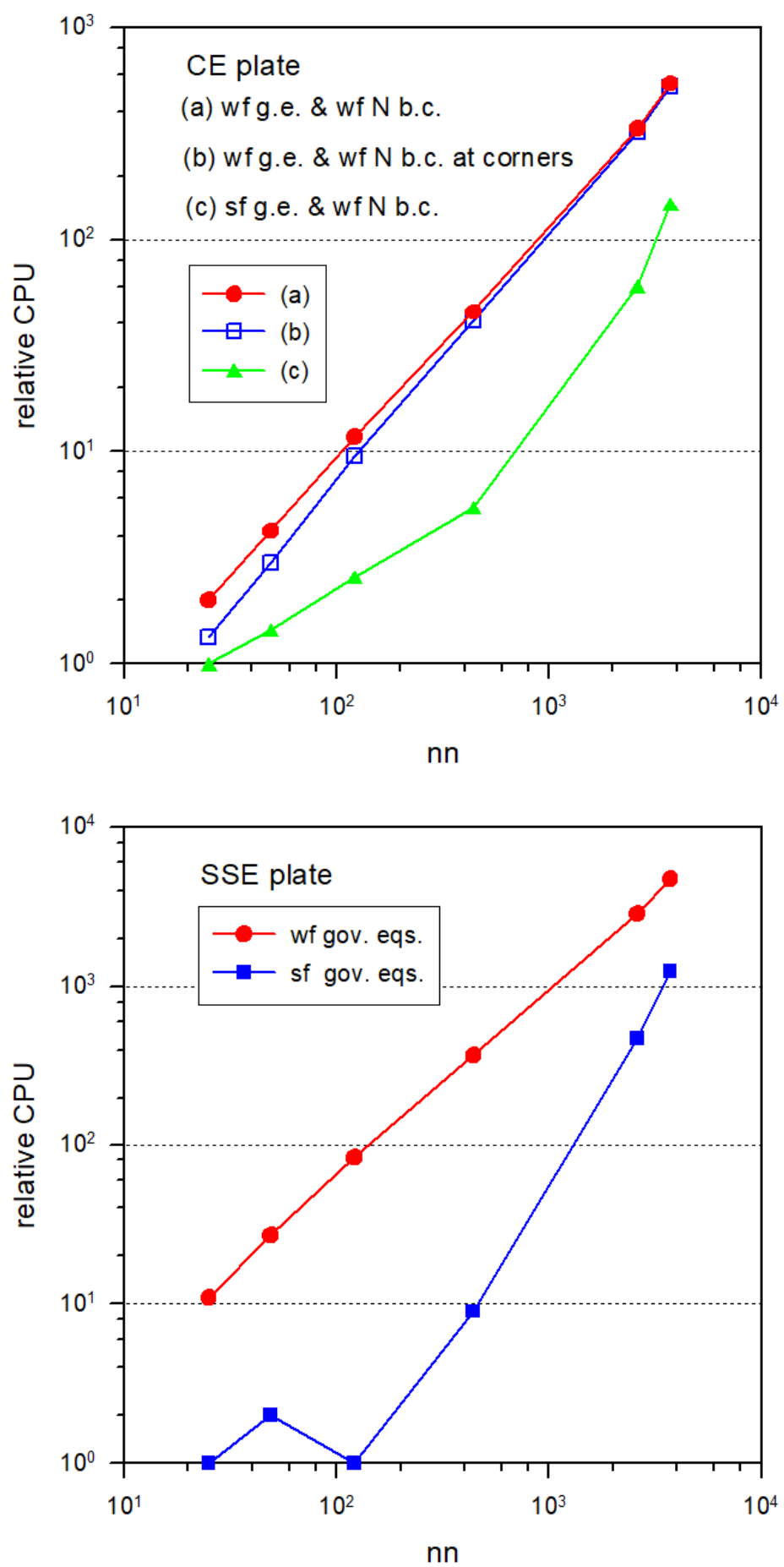

Figure 7: Relative CPU times vs. number of nodes for various techniques in CE and SSE plates. 
Another characteristic of computational methods is the numerical stability, which is determined by the sensitivity of accuracy of numerical solution of discretized equations, i.e. linear system of algebraic equations, with respect to a change of the right hand side evoked for instance by inaccuracy of specification of external influence. For this purpose, the condition number is usually used, defined as the maximum ratio of the relative error in solution to the relative error in the r.h.s. of the system of algebraic equations. The dependences of the condition number on the amount of nodes for CE as well as SSE plates are shown in Fig. 6. Significantly better conditioning of the system of discretized equations is achieved in the SSE plate than in the CE plate.

Finally, for comparison of the computational efficiency of approaches employed in this study, we have used the relative CPU defined as the ratio of the actual CPU measured in seconds and the normalization value. The normalization value is selected as the smallest value of the CPU which is 0.140625 (sec) in case of CE plate and $0.15625(\mathrm{sec})$ in SSE plate with using 25 nodes. From Fig. 7, it can be seen that the numerical integrations involved in weak formulations result in higher values of the CPU than in strong formulations. These differences between the CPU times are decreasing with increasing the number of nodes, since the time needed for solution of the system of discretized equations is becoming dominant and the time needed for numerical integrations is becoming less significant in approaches incorporating weak formulations.

\section{CONCLUSIONS}

The Moving Finite Element approximation has been developed and implemented for the solution of bending boundary value problems considered within the classical Kirchhoff-Love theory. Both the strong and weak formulations for governing equations as well as proper formulations of boundary conditions have been derived and verified in numerical test examples with studying: (i) convergence; (ii) accuracy; (iii) numerical stability of solution of the system of discretized equations; and (iv) computational efficiency of various computational approaches. In numerical studies the dependence on increasing number of nodes is involved.

\section{ACKNOWLEDGEMENT}

The financial support by the Slovak Research and Development Agency through grant SKCN-RD-18-0005 is gratefully acknowledged.

\section{REFERENCES}

[1] Sladek, V., Sladek, J. \& Zhang, C., Local integro-differential equations with domain elements for the numerical solution of partial differential equations with variable coefficients. Jour. Eng. Math., 51, pp. 261-282, 2005.

[2] Sladek, V., Sladek, J. \& Zhang, C., Computation of stresses in non-homogeneous elastic solids by local integral equation method. Comput. Mechanics, 41, pp. 827-845, 2008.

[3] Sladek, V., Sladek, J. \& Zhang, C., The use of finite elements for approximation of field variables on local subdomains in a mesh-free way. Composites with Micro-and NanoStructure, ed. V. Kompis, Springer, pp. 87-106, 2008.

[4] Sladek, V., Repka, M. \& Sladek, J., Moving finite element method. WIT Transactions on Engineering Sciences, vol. 122, WIT Press: Southampton and Boston, 2019. DOI: $10.2495 / \mathrm{BE} 410111$.

[5] Gao, X.W., Gao, L.F., Zhang, Y., Cui, M. \& Lv, J., Free element collocation method: A new method combining advantages of finite element and mesh free methods. Computers and Structures, 215, pp. 10-26, 2019. 
[6] Repka, M., Sladek, V. \& Sladek, J., Numerical study of size effects in micro/nano plates by moving finite elements. Composite Structures, 212, pp. 291-303, 2019.

[7] Sator, L., Sladek, V. \& Sladek, J., Coupling effects in elastic analysis of FGM composite plates by mesh-free methods. Composite Structures, 115, pp. 100-110, 2014.

[8] Timoshenko, S. \& Woinowsky-Krieger, S., Theory of Plates and Shells, McGraw Hill: New York, 1959.

[9] Hughes, T.J.R., The Finite Element Method, Linear Static and Dynamic Finite Element Analysis, Prentice-Hall: Englewood Cliffs, 1987. 